\title{
Uso de clorhexidina y su papel preventivo en las infecciones del torrente sanguíneo asociadas a catéteres en los recién nacidos: revisión de tema
}

\author{
Use of chlorhexidine and its preventive role in \\ catheter-associated bloodstream infections in newborns: a review

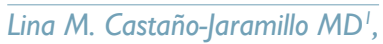 \\ Carolina Henao-Ochoa MD², Ana C. Osorio-Vásquez MD²
}

\begin{abstract}
Resumen: las infecciones asociadas al cuidado de la salud representan un problema de salud pública y son un indicador de la calidad en prestación y gestión en salud. Es por esto que ha sido necesaria la creación de programas de vigilancia y control epidemiológico, y la adaptación e implementación de guías para su prevención. Estas estrategias mejoran la seguridad del paciente y deben ser una prioridad para las instituciones. En este contexto, la infección asociada a catéter es la más común de las infecciones asociadas al cuidado de la salud, principalmente en la población pediátrica, siendo los neonatos los más susceptibles a causa de la inmadurez de sus barreras primarias y sistema inmunológico, y a la alta frecuencia de procedimientos invasivos como los accesos vasculares para la administración de medicamentos o la nutrición parenteral. Actualmente existen múltiples medidas para prevenir dichas infecciones en pediatría y la tendencia es crear estrategias bien estructuradas para facilitar su aplicación. La higiene de manos continúa siendo un pilar fundamental dentro de todos los protocolos para disminuir las tasas de infección asociadas al cuidado de la salud; sin embargo, se requiere de nuevas estrategias para la prevención de las mismas, como el uso de la clorhexidina como antiséptico tópico previo a la inserción de dispositivos intravasculares y el aseo rutinario en pacientes con catéteres centrales, lo cual es el objeto de revisión de este manuscrito.
\end{abstract}

Palabras clave: infección hospitalaria, infecciones relacionadas con catéteres, clorhexidina, recién nacido.

Abstract: Health care-associated infections are a public health concern and reflect healthcare quality. Hereby, it has been necessary to establish an epidemiologic surveillance system and the adaptation and implementation of guidelines for its prevention. These strategies improve safety in health care and must be a priority for all institutions. The most common healthcare-associated infection is catheter-associated bloodstream infection, especially in the pediatric population, with

'Médica, residente de Pediatría, Universidad CES. Medellín, Colombia. Correo electrónico: liniskas@gmail.com ${ }^{2}$ Médica, residente de Pediatría, Universidad CES. Medellín, Colombia.

Conflicto de intereses: las autoras declaran que no tienen conflicto de intereses Medicina \& Laboratorio 2015; 21 : 243-254

Módulo 7 (Microbiología), número 27. Editora Médica Colombiana S.A. $2015^{\odot}$

Recibido el 13 de mayo de 2015; aceptado el 02 de junio de 2015 
the newborns as the most vulnerable group due to immaturity of their immune system and the high rate of invasive procedures such as prolonged vascular access for administration of medication or parenteral nutrition. Nowadays there are many proposals to prevent the infections in pediatrics and the current trend is to create well-structured strategies in order to facilitate their application. Hand's hygiene remains a cornerstone in all the protocols to reduce the health care-associated infections, nevertheless, it requires new strategies to prevention this infections, as chlorhexidine use as a topic antiseptic prior to insertion of intravascular devices and for grooming routine in patients with central venous catheter, which is the reviewing aim of this manuscript.

Key words: Cross infection, catheter-related Infections, chlorhexidine, newborn infant.

Castaño-Jaramillo LM, Henao-Ochoa C, Osorio-Vásquez AC. Uso de clorhexidina y su papel preventivo en las infecciones del torrente sanguíneo asociadas a catéteres en los recién nacidos: revisión de tema. Medicina \& Laboratorio 2015; 21 : 243-254.

L as infecciones asociadas al cuidado de la salud, conocidas anteriormente como infecciones nosocomiales, se han convertido en un tópico de gran interés en la salud pública en los últimos años. Las altas tasas de morbimortalidad a causa de este tipo de infecciones han hecho que su reporte sea obligatorio y que se tomen medidas importantes para su control y prevención. En un intento por ayudar al personal de la salud en esta función, varios organismos internacionales han proporcionado guías de manejo basadas en la evidencia científica para la prevención de las infecciones asociadas al cuidado de la salud [1-3].

En muchas instituciones la implementación de las guías de manejo de las infecciones asociadas al cuidado de la salud ha resultado en un marcado descenso en las tasas de incidencia y mortalidad. Entre 2008 y 2013, los Centros para el Control y la Prevención de Enfermedades (CDC) reportaron una reducción del $46 \%$ al 58\% de las infecciones asociadas a catéter central en las unidades de cuidado intensivo en comparación con las cifras registradas en años anteriores y una disminución hasta de un $8 \%$ en las bacteriemias asociadas a Staphylococcus aureus resistente a la meticilina entre el 2011 y 2013 . Esto representa aproximadamente 25.000 casos menos, 3.000 a 6.000 vidas salvadas y un ahorro de hasta de cuatrocientos millones de dólares [4,5].

La preocupación global alrededor de las infecciones asociadas al cuidado de la salud, sumado al conocimiento de que son infecciones prevenibles y con una alta probabilidad de alcanzar tasas de cero, ha generado una gran presión sobre las Instituciones Prestadoras de Salud (IPS) para reducir al máximo el número de casos [6]. Gracias a los esfuerzos multidisciplinarios y a los recursos invertidos se han alcanzado logros importantes en el tema; sin embargo, todavía existen falencias en cuanto al reporte de las infecciones asociadas al cuidado de la salud en las unidades neonatales, así como la disponibilidad de guías de manejo y prevención basadas en la evidencia y la validación de las mismas a nivel internacional [7]. En esta revisión se presenta la definición y la epidemiología de las infecciones asociadas a catéter, una breve descripción de la población más susceptible a las mismas (los neonatos), el papel y uso de la clorhexidina en la prevención de este tipo de infecciones y las posibles complicaciones asociadas a ésta. 


\section{Definición y epidemiología de las infecciones asociadas a catéteres}

Las infecciones del torrente sanguíneo asociadas a catéter venoso central son aquellas que ocurren durante la permanencia de dicho dispositivo en el paciente o hasta 48 horas después de su retiro [8]. Estas infecciones se presentan en todos los rangos de edad y producen cerca de 90.000 muertes y costos que ascienden a los cuarenta y cinco billones de dólares anuales en los Estados Unidos [9, 10]. La infección asociada a catéter es la más común de las infecciones asociadas al cuidado de la salud en la población pediátrica, la cual se encuentra relacionada con una mayor morbilidad y mortalidad, lo que genera un impacto social y económico negativo para la comunidad [1 |-16].

La tasa de supervivencia reportada para el 2008 por los Centros para el Control y la Prevención de Enfermedades (CDC) para las infecciones asociadas a catéter en los recién nacidos con un peso menor a $750 \mathrm{~g}$ fue de 4,9\% en el caso de los catéteres centrales y del 5,7\% para los catéteres umbilicales. Entretanto, en los neonatos cuyo peso al nacer fue mayor de 2.500 g esta tasa fue del 1,2\% para las infecciones asociadas a catéter central y I,0\% para catéteres umbilicales $[17,18]$.

\section{Los neonatos: la población más susceptible a infecciones asociadas a catéteres}

El período neonatal es el de mayor mortalidad infantil [19,20]. Los neonatos hospitalizados son especialmente susceptibles a las infecciones asociadas al cuidado de la salud, principalmente los prematuros, debido a la inmadurez de sus barreras primarias y su sistema inmunológico, y la alta frecuencia de procedimientos invasivos, como accesos vasculares para la administración de medicamentos o la nutrición parenteral [21,22]. Además, se ha encontrado que los neonatos con menos de $1.500 \mathrm{~g}$ de peso al nacer presentan tres veces más riesgo de adquirir infecciones intrahospitalarias en comparación con los recién nacidos con un peso mayor [23].

La necesidad de establecer un acceso venoso duradero en un vaso de gran calibre ha provocado en los últimos años un aumento en la implementación de catéteres centrales de inserción periférica (PICC; del inglés, Peripherally Inserted Central Catheter), ya que se puede realizar al lado del paciente, con una fácil inserción por el personal de enfermería capacitado y con la viabilidad de la verificación de su posición mediante radiografías [24]. Igualmente, este tipo de catéteres disminuye la necesidad de múltiples punciones venosas, a diferencia del catéter periférico que debe ser cambiado frecuentemente, y permite la administración de sustancias de alta osmolaridad [25,26]. En la población neonatal este tipo de dispositivo ha demostrado ser de larga duración, incluso en recién nacidos de peso extremadamente bajo al nacer $(<1.000 \mathrm{~g})$, sin incrementar el riesgo de complicaciones infecciosas respecto a los catéteres umbilicales [27,28].

Otra opción de acceso central disponible para los pacientes neonatos son los catéteres umbilicales, ya que los vasos en esta zona permanecen permeables durante los primeros días de la vida posnatal. Un cateterismo de vena umbilical puede ser el único recurso en un neonato críticamente enfermo, pero su uso se ve limitado a una o máximo dos semanas de acuerdo a 
las recomendaciones internacionales [29], por tanto, la tendencia actual es mantener el catéter venoso umbilical hasta establecer un acceso central más permanente en los neonatos, teniendo como primera opción un catéter central de inserción periférica; medida que se ha visto asociada con una mayor ganancia de peso y menor tiempo de estancia hospitalaria [30].

\section{Complicaciones infecciosas de los catéteres centrales y su etiología}

A pesar de los múltiples beneficios que ofrecen los catéteres centrales en la población neonatal, tienen potenciales complicaciones a corto y mediano plazo, entre las que se encuentran el desplazamiento accidental, la infección del torrente sanguíneo, la oclusión, la filtración, la flebitis, la fractura del catéter, la trombosis intravascular y el taponamiento cardíaco [3।,32]. Condiciones como la prematuridad, el peso menor a $1.500 \mathrm{~g}$ al nacer, los largos tiempos de permanencia del catéter y la localización no central se asocian con un mayor riesgo de complicaciones [32,33].

La permanencia del catéter central de inserción periférica se ha relacionado directamente con el riesgo de infección del torrente sanguíneo asociada a catéter, con elevación del riesgo en las primeras dos semanas luego de su inserción y permaneciendo elevadas hasta su retiro [3,32]. Por tal razón, algunos autores sugieren considerar una nueva inserción del catéter después de los 35 días, pues después de este período el riesgo de infección asociada a catéter aumenta hasta en un 33\% por día [34].

El sitio de inserción del catéter central de inserción periférica ha sido objeto de algunos estudios. En varias series la posición en las extremidades superiores se ha asociado con un menor número de complicaciones en los recién nacidos pretérmino, por lo que es considerada la primera opción de localización [35,36]. Entretanto, otros autores reportan menores tasas de infección y de colestasis debido a nutrición parenteral total (NPT) prolongada en los pacientes con catéter central de inserción periférica ubicado en las extremidades inferiores. Hasta el momento no hay evidencia definitiva que soporte un sitio de inserción específico, pues en otros estudios no se han encontrado diferencias significativas en la tasa de complicaciones según la localización del catéter, por lo que recomiendan que la decisión se base en al acceso venoso disponible y la preferencia del responsable de la inserción [37,38].

Durante los últimos sesenta años la epidemiología de los microorganismos causantes de las infecciones asociadas al cuidado de la salud del torrente sanguíneo en neonatos ha cambiado drásticamente. En 1950 Staphylococcus aureus era el patógeno más común en los niños hospitalizados. En la década de los sesenta emergieron como relevantes los bacilos Gram negativos, incluyendo Pseudomonas aeruginosa, Klebsiella spp. y Escherichia coli. Desde los años setenta hasta la actualidad predominan los cocos Gram positivos como los microrganismos más importantes, principalmente los estafilococos coagulasa negativa y Staphylococcus aureus, incluyendo las cepas resistentes a la meticilina [36,39]. Cada vez se encuentran con mayor frecuencia microrganismos multirresistentes a causa del uso no controlado de antibióticos de amplio espectro en las unidades neonatales y los cambios secundarios en la microbiota endógena [40]. 
La colonización de las mucosas y la piel por la microbiota endógena ocurre rápidamente luego del nacimiento. Los neonatos que nacen por vía vaginal generalmente se colonizan en su paso por el canal del parto. La mayoría de las veces, estos microorganismos no causan enfermedades graves ni invasivas, pero en los neonatos críticamente enfermos o con muy bajo peso al nacer esta colonización puede llevar a infecciones sistémicas graves y mortales. En los neonatos hospitalizados las superficies de la piel y las mucosas se colonizan más fácilmente por microorganismos como los bacilos Gram negativos, Staphylococcus spp., Enterococcus spp y Candida spp. Estos microorganismos de adquisición intrahospitalaria en muchos casos tienden a ser más virulentos y resistentes a los tratamientos [4I].

Después de los primeros 30 días de inserción del catéter los estafilococos coagulasa negativa representan el microorganismo más importante como agente causal de infección, pero empiezan a obtener mayor relevancia las bacterias Gram negativas [3] y los hongos de las especies de Candida y Malassezia furfur a medida que incrementa el tiempo de permanencia del catéter [42]. La infección del torrente sanguíneo asociada a catéter percutáneo (catéter central de inserción periférica) por estafilococos coagulasa negativa generalmente se da por la colonización intraluminal y se define al aislar el mismo patógeno en el tubo del catéter y en la sangre. En menor medida la infección puede ser dada por colonización extraluminal, demostrada al aislar el mismo microorganismo tanto en la punta del catéter como en la sangre, independiente del microorganismo aislado en el sitio de inserción [43]. Por su parte, la candidiasis neonatal usualmente se relaciona con factores de riesgo que favorecen su aparición como el uso reciente de antibióticos de amplio espectro, específicamente las cefalosporinas de tercera generación, el uso de catéteres percutáneos o la intubación prolongada, la nutrición parenteral con lípidos y la neutropenia [44-46].

Las infecciones asociadas a catéteres centrales usualmente se relacionan con los cuidados durante la implantación y manipulación y el tiempo de permanencia del catéter, así como de los factores individuales de cada neonato $[36,47]$. Aun así, estos dispositivos son fundamentales para el tratamiento, el desarrollo y la sobrevida de los neonatos hospitalizados $[14,25,48]$.

\section{Prevención de las infecciones asociadas a catéteres centrales}

Existen diferentes medidas para prevenir las infecciones asociadas a catéteres centrales. La tendencia actual es utilizar estrategias estructuradas, basadas en paquetes de medidas o modelo bundle que incluyan las disposiciones más apropiadas para la institución en la que van a ser aplicadas, con las que se ha demostrado la reducción en las tasas de infecciones en la población neonatal y la prolongación en la vida media de los accesos intravasculares [49]. Una de las medidas más importantes para prevenir estas infecciones es la evaluación permanente, por parte del personal médico, de la necesidad de inserción de estos dispositivos y la evaluación diaria de las condiciones y pertinencia de continuar con éste [2,3,36].

La higiene de las manos ha demostrado una disminución significativamente alta en las tasas de infección y es parte del protocolo fundamental antes de realizar cualquier tipo de procedimiento estéril invasivo [31,50-52]. También es importante la educación permanente, la retroalimentación del personal, las listas de chequeo durante la inserción y el mantenimiento 
del dispositivo. En muchas instituciones estas medidas han demostrado disminución en las tasas de infección, complicaciones y costos derivados de la atención en salud [2,51,53,54]. En sitios donde estas medidas no han sido suficientes para disminuir las infecciones, se ha propuesto implementar el uso de clorhexidina como antiséptico.

\section{El papel de la clorhexidina en la prevención de las infecciones asociadas a catéteres centrales}

El gluconato de clorhexidina es un antiséptico tópico ampliamente utilizado para la asepsia previa a procedimientos y cirugías, que se encuentra disponible en presentación acuosa o en base de alcohol a diferentes concentraciones y que presenta actividad contra microorganismos Gram positivos, Gram negativos, levaduras y algunos virus. Al tener tan amplio espectro antimicrobiano cubre gran cantidad de los patógenos que amenazan el período neonatal [3,55,56]. La clorhexidina se puede utilizar sola o combinada con alcohol isopropílico [57]. La adición de clorhexidina a las preparaciones a base de alcohol mejora la actividad bactericida por combinación de mecanismos de acción y se logra mayor efecto residual [57,58].

Desde el 2012 la Administración de Medicamentos y Alimentos (FDA; del inglés, Food and Drug Administration) de los Estados Unidos aprobó el uso con precaución de la clorhexidina en menores de dos meses [59]. Además, múltiples reportes a nivel mundial revelan que es el antiséptico de elección para su uso en neonatos con un adecuado perfil de seguridad [ 14,55,60,6 I]. El uso de la clorhexidina en las unidades de cuidados intensivos pediátricas y neonatales se ha incrementado de manera importante en la última década gracias a su impacto sobre las infecciones asociadas a catéter, principalmente cuando se relacionan con las medidas reportadas anteriormente, con un efecto beneficioso sobre la morbimortalidad y el costo de los servicios de salud tanto en países en vía de desarrollo como en los industrializados [51,62-64].

La clorhexidina ha demostrado una menor tasa de colonización bacteriana cualitativa y cuantitativamente en la piel de los neonatos que reciben el baño con clorhexidina en el primer día de vida [56,65,66]; además, disminuye las tasas de hospitalización, sepsis y mortalidad neonatal, principalmente en los recién nacidos pretérmino y de bajo peso al nacer [67-69]. El uso de la clorhexidina, en comparación con otros antisépticos tópicos como las soluciones yodadas, para la asepsia previa a la inserción de catéteres centrales y el uso de gasas impregnadas, ha mostrado una menor tasa de contaminación del catéter y una disminución significativa en el riesgo de infección del catéter central de inserción periférica y de catéteres umbilicales, incluso en neonatos de muy bajo peso al nacer [60,63,64,70-73].

En la población neonatal el uso de la clorhexidina se ve limitado por el temor a las reacciones adversas, principalmente a nivel cutáneo y sistémico. Aunque en ciertos estudios se han encontrado trazas de clorhexidina en la sangre, no se ha asociado con manifestaciones clínicas desfavorables; además, la mayoría de los autores en su publicaciones soportan el uso de la clorhexidina en neonatos, con una adecuada tolerancia, incluso en los recién nacidos prematuros [66,70,74-76]. En los neonatos se ha observado que la limpieza corporal total disminuye transitoriamente la temperatura corporal debido a la inmadurez de los mecanismos para evitar la pérdida de calor; sin embargo, no se han encontrado diferencias significativas entre la disminución de la temperatura corporal luego de la limpieza con la clorhexidina en comparación con 
la solución salina u otros agentes de limpieza utilizados en los recién nacidos, por lo que este parámetro no se considera una limitante para su uso [56,66,74].

Por otra parte, se han descrito quemaduras asociadas a clorhexidina con las preparaciones a base de alcohol isopropílico al 70\%, sin establecer con claridad si la causa de esta reacción cutánea es a la clorhexidina como tal o al diluyente que se encuentra en una alta concentración $[61,77]$. Con la clorhexidina acuosa como único agente hasta nuestro conocimiento solo se encuentra disponible un reporte en la literatura de quemaduras en la piel en un recién nacido pretérmino extremo, gemelar, de 25 semanas de vida, en quién se utilizó clorhexidina como asepsia previo al cateterismo venoso umbilical en las primeras horas de vida [78].

La limpieza con las toallas de clorhexidina acuosa al $2 \%$ ha mostrado un gran beneficio para la disminución de infecciones asociadas al cuidado de la salud y del torrente sanguíneo asociadas a catéter central; además, una reducción de la prevalencia y transmisión de microorganismos multirresistentes en unidades de cuidado intensivo tanto de adultos como pediátricas [79-8I]. No obstante, la evidencia del uso de la limpieza con toallas de clorhexidina en los neonatos es limitada y casi todos los reportes disponibles provienen de la evaluación en la población adulta.

\section{Experiencias del uso de clorhexidina}

En 1991, Maki y colaboradores [82] reportaron que la preparación del sitio de inserción del catéter venoso central con clorhexidina disminuía las tasas de infección local en comparación con el alcohol al 70\% y la povidona yodada al 10\% ( $p=0,02)$. En un metanálisis realizado por Chaiyakunapruk y colaboradores 2002 [72], que incluía ocho estudios que involucraban a 4. 143 pacientes con catéteres de varios tipos, se comparó el gluconato de clorhexidina con la povidona yodada para el cuidado y la antisepsia del sitio de inserción del catéter. El resultado fue una reducción del riesgo de bacteriemia (riesgo relativo $=0,49$; intervalo de confianza del $95 \%=0,28$ a 0,88 y reducción de las tasas de bacteriemia asociada a catéter vascular central (riesgo relativo $=0,5 \mathrm{I}$; intervalo de confianza del 95\%=0,27 a 0,97), cuando se usaba gluconato de clorhexidina en lugar de povidona yodada.

Valles y colaboradores (2008) [83] compararon tres soluciones antisépticas para prevenir la colonización de los catéteres en una unidad de cuidados intensivos de adultos: la povidona yodada acuosa al 10\%, el gluconato de clorhexidina acuosa al $2 \%$ y el gluconato de clorhexidina alcohólica al 0,5\%, para la antisepsia cutánea de 63I pacientes con catéteres vasculares. Los autores encontraron que la incidencia de colonización del catéter fue significativamente menor en el grupo del gluconato de clorhexidina alcohólica en comparación con el de povidona yodada ( $14,2 \%$ frente a $24,7 \%$; riesgo relativo $=0,5$; intervalo de confianza del $95 \%=0,3$ a 0,8$)$, y en el grupo del gluconato de clorhexidina acuosa respecto al grupo de la povidona yodada ( $16,1 \%$ frente a 24,7\%; riesgo relativo=0,6; intervalo de confianza del $95 \%=0,4$ a 0,9$)$. Por otro lado, no se encontraron diferencias significativas entre los dos grupos de gluconato de clorhexidina. A pesar de estos resultados, no se encontraron diferencias estadísticamente significativas en la incidencia de bacteriemia para los tres grupos $(p \geq 0,20)$.

Rupp y colaboradores (2005) [84] reportaron que los catéteres impregnados en su exterior con clorhexidina y sulfadiazina presentan tasas menores de colonización $(p<0,01)$ y tendencia 
a tasas menores de bacteriemia $(p=0,06)$ en comparación con los catéteres sin impregnar. Aunque no se les puede atribuir el efecto sólo a la clorhexidina o a la sulfadiazina de manera independiente, estos resultados representan hallazgos que ameritan ser estudiados con mayor profundidad. Por su parte, Small y colaboradores (2008) [85] encontraron que el uso de una solución de gluconato de clorhexidina al $2 \%$ en alcohol isopropílico al $70 \%$ para desinfectar la piel antes de la venopunción reduce el número de catéteres periféricos colonizados y contaminados en comparación con el uso solamente de una solución alcohólica $(p<0,00$ I).

Por otro lado, Karpanen y colaboradores (201 I) [86] realizaron un estudio in vitro con apósitos para catéteres vasculares impregnados con gel de clorhexidina, en el que encontraron que después de la inoculación de 6 log de unidades formadoras de colonias (UFC) de Staphylococcus aureus resistente a la meticilina en una muestra de piel de donantes cubierta con estos apósitos no se detectan bacterias después de 24 horas; incluso, la actividad antimicrobiana de estos apósitos se pudo observar hasta 7 días después de la aplicación.

Otra estrategia que se ha utilizado para prevenir las infecciones asociadas a la atención en salud es el lavado diario de los pacientes con clorhexidina al $2 \%$, el cual ha demostrado ser efectivo para reducir las tasas de bacteriemia asociada a los catéteres venosos centrales. En un estudio cuasi experimental llevado a cabo por Munoz-Price y colaboradores (2009) [87], en una institución de cuidados prolongados, se encontró una reducción del 99\% en las tasas de bacteriemia asociada a catéteres venosos centrales cuando se implementó el lavado diario con clorhexidina. De forma similar, Popovich y colaboradores (2009) [88], en una unidad de cuidados intensivos, observaron que, después de implementar el lavado diario con clorhexidina al $2 \%$ sin enjuagar, se reducía significativamente la bacteriemia asociada al catéter venoso central, pasando de 5,3 I a 0,69 casos por cada $1.000(p=0,006)$.

Mediante modelos de toma de decisiones se ha concluido que, aunque el gluconato de clorhexidina tiene mayor valor comercial que la povidona yodada, al reducir la incidencia de bacteriemias asociadas al catéter disminuye los costos hospitalarios totales hasta en US\$I I 3 por catéter [89].

En Canadá, Quach y colaboradores (2014) [22] elaboraron un estudio en recién nacidos con catéter venoso central, con análisis estadísticos ajustados, en el cual mostraron una disminución del 77\% del riesgo de infección asociada a catéter con la intervención del baño con clorhexidina respecto al baño tradicional con un jabón suave (riesgo relativo=0,33; intervalo de confianza del 95\%=0, 15 a 0,73) en los recién nacidos con peso mayor de 1.000 g. Por su parte, los recién nacidos que debido a su peso (menor o igual a $1.000 \mathrm{~g}$ ) o edad (menor a 28 días) no fueron sometidos a la intervención con el baño de clorhexidina no presentaron cambios significativos en las tasas de bacteriemia asociada a catéter, manteniendo los niveles de infección. Por otro lado, no se registraron reacciones adversas con el uso de la clorhexidina. Lo anterior sugiere que la limpieza con toallas de clorhexidina puede ser la responsable de la reducción en las tasas de infección asociada a catéter en neonatos sin producir efectos secundarios desfavorables.

Según el protocolo utilizado en el estudio de Quach y colaboradores (2014) [22], se recomienda el uso de toallas de clorhexidina acuosa para la limpieza corporal en neonatos con dispositivos intravasculares para reducir la tasa de infección asociada a catéter de la siguiente manera: a) en los recién nacidos con un peso mayor a 1.000 g y con más de 28 semanas de edad gestacional al momento del parto, desde el nacimiento y con una frecuencia interdiaria, b) 
en los recién nacidos con peso extremadamente bajo al nacer $(<1.000 \mathrm{~g})$ o con menos de 28 semanas de edad gestacional, después del día 28 de vida, para disminuir el riesgo de reacciones adversas que se han descrito excepcionalmente en estos pacientes, con una frecuencia de dos veces por semana, y c) en los recién nacidos independiente de su peso al nacer y con más de 36 semanas de edad gestacional se puede realizar diariamente.

De acuerdo con los hallazgos anteriores, las guías para la prevención de infecciones asociadas a los catéteres intravasculares de los Centros para el Control y la Prevención de Enfermedades (CDC), las guías de práctica clínica para el uso de antibióticos en pacientes neutropénicos con cáncer y las guías de práctica clínica para la prevención de infecciones hospitalarias asociadas al uso de dispositivos médicos de la Asociación Colombiana de Infectología, recomiendan el uso de una solución a base de clorhexidina para la antisepsia cutánea antes de la inserción de un catéter central (recomendación A-I) [90,9l].

\section{Conclusión}

Las infecciones asociadas al cuidado de la salud, incluyendo las infecciones del torrente sanguíneo asociadas a catéter, representan problemas de salud pública que influyen en la morbilidad y mortalidad de los pacientes, y en las mayores estancias hospitalarias y costos en los servicios de salud. La clorhexidina tópica ha demostrado su beneficio para reducir las infecciones asociadas a catéteres en la población adulta y pediátrica. En la última década se ha incrementado el uso de la clorhexidina en las unidades de cuidado intensivo neonatal para reducir las tasas de colonización bacteriana en la piel de los recién nacidos y con ello reducir las infecciones del torrente sanguíneo en esta población tan susceptible.

Aún se requieren más estudios para evaluar la seguridad del uso de la clorhexidina, principalmente en los recién nacidos pretérmino extremos debido al riesgo de reacciones cutáneas locales y absorción sistémica; no obstante, las experiencias de algunos grupos de investigación muestran una adecuada tolerancia que posibilita su uso para la antisepsia cutánea antes de la inserción de un catéter central para prevenir las posibles infecciones asociadas.

Es así como los hallazgos en el estudio cuasiexperimental realizado en el hospital infantil de Montreal por Quach y colaboradores [22], es el punto de partida para recomendaciones futuras. En su protocolo el uso de toallas de clorhexidina acuosa al $2 \%$, para la asepsia del paciente en vez del uso de jabón, sin retirar la clorhexidina después del baño y dependiendo de la edad gestacional, han mostrado ser seguras y efectivas en el control de la infección asociada a catéter.

\section{Bibliografía}

I. Gould CV, Umscheid CA, Agarwal RK, Kuntz G, Pegues DA. Guideline for prevention of catheter-associated urinary tract infections 2009. Infect Control Hosp Epidemiol 2010; 31: 319-326.

2. Fisher D, Cochran KM, Provost LP, Patterson J, Bristol $\mathrm{T}$, Metzguer K, et al. Reducing central line-associated bloodstream infections in North Carolina NICUs. Pediatrics 2013; I32: el664-167|.

3. Milstone AM, Reich NG, Advani S, Yuan G, Bryant K,

Coffin SE, et al. Catheter dwell time and CLABSIs in neonates with PICCs: a multicenter cohort study. Pediatrics 2013; 132: el609-1615.

4. Centers for Disease Control and Prevention (CDC). Vital signs: central line-associated blood stream infections--United States, 200I, 2008, and 2009. MMWR Morb Mortal Wkly Rep 20I I; 60: 243-248.

5. Centers for Disease Control and Prevention (CDC). National and State Healthcare-Associated Infections 
Progress Report. Atlanta, Estados Unidos: National Center for Emerging and Zoonotic Infectious Diseases. 2015. Disponible: http://www.cdc.gov/HAl/pdfs/progress-report/hai-progress-report-20 I5.pdf. Consultado: abr 2015.

6. Fanaroff AA, Hack M, Walsh MC. The NICHD neonatal research network: changes in practice and outcomes during the first I5 years. Semin Perinatol 2003; 27: 28I-287.

7. Gaynes RP, Edwards JR, Jarvis WR, Culver DH, Tolson JS, Martone WJ. Nosocomial infections among neonates in high-risk nurseries in the United States. National Nosocomial Infections Surveillance System. Pediatrics 1996; 98: 357-361.

8. Centers for Disease Control and Prevention (CDC). The National Healthcare Safety Network (NHSN) Manual: Patient safety component protocol. Atlanta, Estados Unidos: Division of Healthcare Quality Promotion, National Center for Infectious Diseases. 2008. Disponible: http:// mww.dhcs.ca.gov/provgovpart/initiatives/nqi/Documents/ NHSNManPSPCurr.pdf. Consultado: abr 2015.

9. Stone PW. Economic burden of healthcare-associated infections: an American perspective. Expert Rev Pharmacoecon Outcomes Res 2009; 9: 417-422.

10. Centers for Disease Control (CDC). Public health focus: surveillance, prevention, and control of nosocomial infections. MMWR Morb Mortal Wkly Rep 1992; 4I: 783-787.

II. Díaz Guio DA, Montoya Navarrete F. Prevención de la infección nosocomial en UCl. Acta Colomb Cuid Intensivo 201 I; II: 68-75.

12. Becerra MR, Tantalean JA, Suarez VJ, Alvarado MC, Candela JL, Urcia FC. Epidemiologic surveillance of nosocomial infections in a Pediatric Intensive Care Unit of a developing country. BMC Pediatr 20 10; 10: 66.

13. van der Zwet WC, Kaiser AM, van Elburg RM, Berkhof J, Fetter WP, Parlevliet GA, et al. Nosocomial infections in a Dutch neonatal intensive care unit: surveillance study with definitions for infection specifically adapted for neonates. J Hosp Infect 2005; 61: 300-3II.

14. Johann DA, De Lazzari LS, Pedrolo E, Mingorance P, de Almeida TQ, Danski MT. [Peripherally inserted central catheter care in neonates: an integrative literature review]. Rev Esc Enferm USP 2012; 46: I503-I5II.

I5. Johnson TJ, Patel AL, Jegier BJ, Engstrom JL, Meier PP. Cost of morbidities in very low birth weight infants. J Pediatr 20।3; 162: 243-249 e24I.

16. Parker LA, Krueger C, Sullivan S, Kelechi T, Mueller M. Effect of breast milk on hospital costs and length of stay among very low-birth-weight infants in the NICU. Adv Neonatal Care 20 I2; 12: 254-259.

17. Edwards JR, Peterson KD, Mu Y, Banerjee S, Allen-Bridson K, Morrell G, et al. National Healthcare Safety Network (NHSN) report: data summary for 2006 through 2008, issued December 2009. Am J Infect Control 2009; 37: 783-805.

18. Dudeck MA, Horan TC, Peterson KD, Allen-Bridson K, Morrell G, Anttila A, et al. National Healthcare Safety Network report, data summary for 20I I, device-associated module. Am J Infect Control 2013; 41: 286-300.

19. Lawn JE, Cousens S, Zupan J. 4 million neonatal deaths: when? Where? Why? Lancet 2005; 365: 891-900.

20. Alkiaat A, Hutchinson M, Jacques A, Sharp MJ, Dickinson JE. Evaluation of the frequency and obstetric risk factors associated with term neonatal admissions to special care units. Aust N Z J Obstet Gynaecol 20 I3; 53: 277-282.

21. Brodie SB, Sands KE, Gray JE, Parker RA, Goldmann DA, Davis RB, et al. Occurrence of nosocomial bloodstream infections in six neonatal intensive care units. Pediatr Infect Dis J 2000; 19: 56-65.

22. Quach C, Milstone AM, Perpete C, Bonenfant M, Moore $\mathrm{DL}$, Perreault T. Chlorhexidine bathing in a tertiary care neonatal intensive care unit: impact on central line-associated bloodstream infections. Infect Control Hosp Epidemiol 20|4; 35: |58-| 63.

23. Krause PJ, Herson VC, Boutin-Lebowitz J, Eisenfeld L, Block C, LoBello T, et al. Polymorphonuclear leukocyte adherence and chemotaxis in stressed and healthy neonates. Pediatr Res 1986; 20: 296-300.

24. Martínez R, Sanchez R, Villanueva Y, Gallón C. Utilidad del catéter central de acceso periférico (PICC) en recién nacidos prematuros de la unidad de cuidado intensivo neonatal de la Clínica General del Norte. Rev Colomb Enferm 20।2; 7: 22-30.

25. Ainsworth SB, Clerihew L, McGuire W. Percutaneous central venous catheters versus peripheral cannulae for delivery of parenteral nutrition in neonates. Cochrane Database Syst Rev 2007: CD004219.

26. McCay AS, Elliott EC, Walden M. Videos in clinical medicine. PICC placement in the neonate. $N$ Engl J Med 2014: 370: el7.

27. Ozkiraz S, Gokmen Z, Anuk Ince D, Akcan AB, Kilicdag $H$, Ozel $D$, et al. Peripherally inserted central venous catheters in critically ill premature neonates. J Vasc Access 2013; 14: 320-324.

28. Arnts IJ, Bullens LM, Groenewoud JM, Liem KD. Comparison of complication rates between umbilical and peripherally inserted central venous catheters in newborns. J Obstet Gynecol Neonatal Nurs 20 I4; 43: 205-2I 5.

29. Butler-O'Hara M, D'Angio CT, Hoey H, Stevens TP. An evidence-based catheter bundle alters central venous catheter strategy in newbom infants. J Pediatr 2012; 160: 972-977 e972

30. Yang ZM, Wang SN, Ma YL, Yang XL, Zhang WY, Zhu MY. [Application of umbilical venous catheter combined with peripherally inserted central catheter in very low birth weight infants]. Zhongguo Dang Dai Er Ke Za Zhi 2013; 15: 353-355.

31. Ohki Y, Maruyama K, Harigaya A, Kohno M, Arakawa $\mathrm{H}$. Complications of peripherally inserted central venous catheter in Japanese neonatal intensive care units. Pediatr Int 2013; 55: 185-189.

32. Duarte ED, Pimenta AM, e Silva BC, de Paula CM. [Factors associated with infection from the use of peripherally inserted central catheters in a neonatal intensive care unit]. Rev Esc Enferm USP 20 I3; 47: 547-554.

33. Jumani K, Advani S, Reich NG, Gosey L, Milstone AM. Risk factors for peripherally inserted central venous catheter complications in children. JAMA Pediatr 2013; 167: $429-435$. 
34. Sengupta A, Lehmann C, Diener-West M, Perl TM, Milstone AM. Catheter duration and risk of CLA-BSI in neonates with PICCs. Pediatrics 20 I0; I25: 648-653.

35. Panagiotounakou P, Antonogeorgos G, Gounari E, Papadakis S, Labadaridis J, Gounaris AK. Peripherally inserted central venous catheters: frequency of complications in premature newborn depends on the insertion site. J Perinatol 20।4; 34: 46|-463.

36. Corzine M, Willett LD. Neonatal PICC: one unit's sixyear experience with limiting catheter complications. Neonatal Netw 2010; 29: 161-173.

37. Wrightson DD. Peripherally inserted central catheter complications in neonates with upper versus lower extremity insertion sites. Adv Neonatal Care 2013; 13 : 198-204

38. Bulbul A, Okan F, Nuhoglu A. Percutaneously inserted central catheters in the newborns: a center's experience in Turkey. J Matern Fetal Neonatal Med 20 I0; 23: 529-535.

39. Polin RA, Saiman L. Nosocomial Infections in the Neonatal Intensive Care Unit. NeoReviews 2003; 4: e8 I-e89.

40. Helwich E, Wojkowska-Mach J, Borszewska-Kornacka M, Gadzinowski J, Gulczynska E, Kordek A, et al. [Epidemiology of infections in very low birth weight infants. Polish Neonatology Network research]. Med Wieku Rozwoj 2013; 17: 224-231.

41. Polin RA, Denson S, Brady MT. Epidemiology and diagnosis of health care-associated infections in the NICU. Pediatrics 2012; 129: el 104-1 109.

42. Sohn AH, Garrett DO, Sinkowitz-Cochran RL, Grohskopf LA, Levine GL, Stover BH, et al. Prevalence of nosocomial infections in neonatal intensive care unit patients: Results from the first national point-prevalence survey. | Pediatr 200 I; 139: 821-827.

43. Garland JS, Alex CP, Sevallius JM, Murphy DM, Good MJ, Volberding AM, et al. Cohort study of the pathogenesis and molecular epidemiology of catheter-related bloodstream infection in neonates with peripherally inserted central venous catheters. Infect Control Hosp Epidemiol 2008; 29: 243-249.

44. Yu Y, Du L, Yuan T, Zheng J, Chen A, Chen L, et al. Risk factors and clinical analysis for invasive fungal infection in neonatal intensive care unit patients. Am J Perinatol 2013; 30: 589-594.

45. Benjamin DK, Jr., Ross K, McKinney RE, Jr., Benjamin DK, Auten R, Fisher RG. When to suspect fungal infection in neonates: A clinical comparison of Candida albicans and Candida parapsilosis fungemia with coagulase-negative staphylococcal bacteremia. Pediatrics 2000; 106: 712-7|8.

46. Saiman L, Ludington E, Pfaller M, Rangel-Frausto S, Wiblin RT, Dawson J, et al. Risk factors for candidemia in Neonatal Intensive Care Unit patients. The National Epidemiology of Mycosis Survey study group. Pediatr Infect Dis J 2000; 19: 319-324.

47. Linares J, Sitges-Serra A, Garau J, Perez JL, Martin R. Pathogenesis of catheter sepsis: a prospective study with quantitative and semiquantitative cultures of catheter hub and segments. J Clin Microbiol 1985; 21: 357-360.

48. Klein JF, Shahrivar F. Use of percutaneous silastic central venous catheters in neonates and the management of infectious complications. Am J Perinatol 1992; 9: 26I-264.
49. Boutaric E, Gilardi M, Cecile W, Flechelles O. [lmpact of clinical practice guidelines on the incidence of bloodstream infections related to peripherally inserted central venous catheter in preterm infants]. Arch Pediatr 2013; 20: 130-136.

50. Garland JS, Uhing MR. Strategies to prevent bacterial and fungal infection in the neonatal intensive care unit. Clin Perinatol 2009; 36: 1-13.

5I. Jeong IS, Park SM, Lee JM, Song JY, Lee SJ. Effect of central line bundle on central line-associated bloodstream infections in intensive care units. Am J Infect Control 2013; 4I: 710-716.

52. Graham PL, 3rd. Simple strategies to reduce healthcare associated infections in the neonatal intensive care unit: line, tube, and hand hygiene. Clin Perinatol 20 I0; 37: 645-653.

53. Ceballos K, Waterman K, Hulett T, Makic MB. Nurse-driven quality improvement interventions to reduce hospital-acquired infection in the NICU. Adv Neonatal Care 2013; I3: 154- |63; quiz I64- 155.

54. Bizzarro MJ, Sabo B, Noonan M, Bonfiglio MP, Northrup V, Diefenbach K. A quality improvement initiative to reduce central line-associated bloodstream infections in a neonatal intensive care unit. Infect Control Hosp Epidemiol 20I0; 31: 24I-248.

55. Tamma PD, Aucott SW, Milstone AM. Chlorhexidine use in the neonatal intensive care unit: results from a national survey. Infect Control Hosp Epidemiol 20 I0; 31 : 846-849.

56. Darmstadt GL, Hossain MM, Choi Y, Shirin M, Mullany LC, Islam M, et al. Safety and effect of chlorhexidine skin cleansing on skin flora of neonates in Bangladesh. Pediatr Infect Dis J 2007; 26: 492-495.

57. O’Donnell JA, Gelone SP. Topical Antibacterials. En: Mandell GL, Bennett JE, Dolin R, eds. Mandell, Douglas, and Bennett's Principles and Practice of Infectious Diseases. Vol. I (ed 7ma). Filadelfia, Estados Unidos: Churchill Livingstone, Elsevier, 2010: 521-532.

58. Hibbard JS, Mulberry GK, Brady AR. A clinical study comparing the skin antisepsis and safety of ChloraPrep, $70 \%$ isopropyl alcohol, and $2 \%$ aqueous chlorhexidine. J Infus Nurs 2002; 25: 244-249.

59. U.S. Food and Drug Administration. FDA Approved Drug Products. Maryland, Estados Unidos. Disponible: http://www.accessdata.fda.gov/scripts/cder/drugsatfda/ index.cfm?fuseaction=Search.SearchAction\&SearchType=BasicSearch\&SearchTerm $=$ CHLORHEXIDINE. Consultado: abr 2014.

60. Sharpe E, Pettit J, Ellsbury DL. A national survey of neonatal peripherally inserted central catheter (PICC) practices. Adv Neonatal Care 20I3; 13: 55-74.

6I. Upadhyayula S, Kambalapalli M, Harrison CJ. Safety of anti-infective agents for skin preparation in premature infants. Arch Dis Child 2007; 92: 646-647.

62. Suresh GK, Edwards WH. Central line-associated bloodstream infections in neonatal intensive care: changing the mental model from inevitability to preventability. Am J Perinatol 2012; 29: 57-64.

63. Sannoh S, Clones B, Munoz J, Montecalvo M, Parvez B. A multimodal approach to central venous catheter hub care can decrease catheter-related bloodstream infec- 
tion. Am J Infect Control 20 I0; 38: 424-429.

64. Curry S, Honeycutt M, Goins G, Gilliam C. Catheter-associated bloodstream infections in the NICU: getting to zero. Neonatal Netw 2009; 28: I 5 I- 155.

65. Da Cunha ML, Procianoy RS, Franceschini DT, De Oliveira LL, Cunha ML. Effect of the first bath with chlorhexidine on skin colonization with Staphylococcus aureus in normal healthy term newborns. Scand I Infect Dis 2008; 40: 615-620.

66. Mullany LC, Khatry SK, Sherchand JB, LeClerq SC, Darmstadt GL, Katz J, et al. A randomized controlled trial of the impact of chlorhexidine skin cleansing on bacterial colonization of hospital-born infants in Nepal. Pediatr Infect Dis J 2008; 27: 505-5II.

67. Rosenthal VD. Central line-associated bloodstream infections in limited-resource countries: a review of the literature. Clin Infect Dis 2009; 49: 1899-1907.

68. Taha TE, Biggar RJ, Broadhead RL, Mtimavalye LA, Justesen $A B$, Liomba GN, et al. Effect of cleansing the birth canal with antiseptic solution on maternal and newborn morbidity and mortality in Malawi: clinical trial. BMJ 1997; 315: 216-219; discussion 220.

69. Tielsch JM, Darmstadt GL, Mullany LC, Khatry SK, Katz J, LeClerq SC, et al. Impact of newborn skin-cleansing with chlorhexidine on neonatal mortality in southern Nepal: a community-based, cluster-randomized trial. Pediatrics 2007; I 19: e330-340.

70. Nuntnarumit P, Sangsuksawang N. A randomized controlled trial of $1 \%$ aqueous chlorhexidine gluconate compared with 10\% povidone-iodine for topical antiseptic in neonates: effects on blood culture contamination rates. Infect Control Hosp Epidemiol 20 I3; 34: 430-432.

7I. Atahan K, Cokmez A, Bekoglu M, Durak E, Tavusbay C, Tarcan E. The effect of antiseptic solution in central venous catheter care. Bratisl Lek Listy 20 I 2; I | 3: 548-55 |.

72. Chaiyakunapruk N, Veenstra DL, Lipsky BA, Saint S Chlorhexidine compared with povidone-iodine solution for vascular catheter-site care: a meta-analysis. Ann Intern Med 2002; I 36: 792-80 |

73. Garland JS, Alex CP, Mueller CD, Otten D, Shivpuri C, Harris MC, et al. A randomized trial comparing povidone-iodine to a chlorhexidine gluconate-impregnated dressing for prevention of central venous catheter infections in neonates. Pediatrics 200 I; 107: |431-1436.

74. Pereira L, Chipato T, Mashu A, Mushangwe V, Rusakaniko S, Bangdiwala SI, et al. Randomized study of vaginal and neonatal cleansing with $1 \%$ chlorhexidine. Int J Gynaecol Obstet 20 I I; I I 2: 234-238.

75. Wilson CM, Gray G, Read JS, Mwatha A, Lala S, Johnson S, et al. Tolerance and safety of different concentrations of chlorhexidine for peripartum vaginal and infant washes: HIVNET 025. J Acquir Immune Defic Syndr 2004; 35: I38- 43.

76. Garland JS, Alex CP, Uhing MR, Peterside IE, Rentz A, Harris MC. Pilot trial to compare tolerance of chlorhexidine gluconate to povidone-iodine antisepsis for central venous catheter placement in neonates. J Perinatol 2009; 29: 808-813.

77. Reynolds PR, Banerjee S, Meek JH. Alcohol burns in extremely low birthweight infants: still occurring. Arch Dis Child Fetal Neonatal Ed 2005; 90: FIO.
78. Lashkari HP, Chow P, Godambe S. Aqueous $2 \%$ chlorhexidine-induced chemical bums in an extremely premature infant. Arch Dis Child Fetal Neonatal Ed 20 12; 97: F64.

79. Climo MW, Yokoe DS, Warren DK, Perl TM, Bolon M, Herwaldt LA, et al. Effect of daily chlorhexidine bathing on hospital-acquired infection. N Engl J Med 20 I3; 368: 533-542.

80. O'Horo JC, Silva GL, Munoz-Price LS, Safdar N. The efficacy of daily bathing with chlorhexidine for reducing healthcare-associated bloodstream infections: a meta-analysis. Infect Control Hosp Epidemiol 20 I2; 33: 257-267.

8I. Viray MA, Morley JC, Coopersmith CM, Kollef MH, Fraser VJ, Warren DK. Daily bathing with chlorhexidine-based soap and the prevention of Staphylococcus aureus transmission and infection. Infect Control Hosp Epidemiol 20।4; 35: 243-250.

82. Maki DG, Ringer M, Alvarado CJ. Prospective randomised trial of povidone-iodine, alcohol, and chlorhexidine for prevention of infection associated with central venous and arterial catheters. Lancet 1991; 338: 339-343.

83. Valles J, Fernandez I, Alcaraz D, Chacon E, Cazorla A, Canals $M$, et al. Prospective randomized trial of 3 antiseptic solutions for prevention of catheter colonization in an intensive care unit for adult patients. Infect Control Hosp Epidemiol 2008; 29: 847-853.

84. Rupp ME, Lisco SJ, Lipsett PA, Perl TM, Keating K, Civetta JM, et al. Effect of a second-generation venous catheter impregnated with chlorhexidine and silver sulfadiazine on central catheter-related infections: a randomized, controlled trial. Ann Intern Med 2005; I43: 570-580.

85. Small H, Adams D, Casey AL, Crosby CT, Lambert PA, Elliott T. Efficacy of adding 2\% (w/v) chlorhexidine gluconate to $70 \%(\mathrm{v} / \mathrm{v})$ isopropyl alcohol for skin disinfection prior to peripheral venous cannulation. Infect Control Hosp Epidemiol 2008; 29: 963-965.

86. Karpanen TJ, Casey AL, Conway BR, Lambert PA, Elliott TS. Antimicrobial activity of a chlorhexidine intravascular catheter site gel dressing. J Antimicrob Chemother 20 I 1; 66: I777- 1784.

87. Munoz-Price LS, Hota B, Stemer A, Weinstein RA. Prevention of bloodstream infections by use of dally chlorhexidine baths for patients at a long-term acute care hospital. Infect Control Hosp Epidemiol 2009; 30: 1031-1035.

88. Popovich KJ, Hota B, Hayes R, Weinstein RA, Hayden MK. Effectiveness of routine patient cleansing with chlor hexidine gluconate for infection prevention in the medical intensive care unit. Infect Control Hosp Epidemiol 2009; 30: 959-963.

89. Chaiyakunapruk N, Veenstra DL, Lipsky BA, Sullivan SD, Saint S. Vascular catheter site care: the clinical and economic benefits of chlorhexidine gluconate compared with povidone iodine. Clin Infect Dis 2003; 37: 764-77I.

90. O'Grady N P, Alexander M, Dellinger EP, Gerberding JL, Heard SO, Maki DG, et al. Guidelines for the prevention of intravascular catheter-related infections. Am J Infect Control 2002; 30: 476-489.

91. Álvarez CA, Cortés JA, Gómez $\mathrm{CH}$, Fernández JA Sossa MP, Beltrán F, et al. Guías de práctica clínica para la prevención de infecciones intrahospitalarias asociadas al uso de dispositivos médicos. Infectio 2010; 14: 292-308. 